\title{
Long-Term In Vitro System for Maintenance and Amplification of Root-Knot Nematodes in Cucumis sativus Roots
}

\author{
Fernando E. Díaz-Manzano', Rocío Olmo ${ }^{+}$, Javier Cabrera*, Marta Barcala, \\ Carolina Escobar* and Carmen Fenoll \\ Área de Fisiología Vegetal, Facultad de Ciencias Ambientales y Bioquímica, Universidad de Castilla-La Mancha, Toledo, \\ Spain
}

Root-knot nematodes $(\mathrm{RKN})$ are polyphagous plant-parasitic roundworms that produce large crop losses, representing a relevant agricultural pest worldwide. After

OPEN ACCESS

Edited by:

Andrea Genre,

University of Turin, Italy

Reviewed by:

Janice De Almeida Engler, Institut National de la Recherche

Agronomique, France

Krzysztof Wieczorek,

University of Natural Resources and Life Sciences, Vienna, Austria

${ }^{*}$ Correspondence:

Carolina Escobar

carolina.escobar@uclm.es; Javier Cabrera

javier.cabrerachaves@uclm.es

tThese authors have contributed equally to this work.

Specialty section: This article was submitted to Plant Biotic Interactions, a section of the journal

Frontiers in Plant Science

Received: 30 October 2015

Accepted: 22 January 2016

Published: 22 February 2016

Citation:

Díaz-Manzano FE, Olmo R, Cabrera J, Barcala M, Escobar C and Fenoll C (2016) Long-Term In Vitro System for Maintenance and Amplification of Root-Knot Nematodes in Cucumis sativus Roots. Front. Plant Sci. 7:124. doi: 10.3389/fpls.2016.00124 infection, they induce swollen root structures called galls containing giant cells (GCs) indispensable for nematode development. Among efficient control methods are biotechnology-based strategies that require a deep knowledge of underlying molecular processes during the plant-nematode interaction. Methods of achieving this knowledge include the application of molecular biology techniques such as transcriptomics (as massive sequencing or microarray hybridization), proteomics or metabolomics. These require aseptic experimental conditions, as undetected contamination with other microorganisms could compromise the interpretation of the results. Herein, we present a simple, efficient and long-term method for nematode amplification on cucumber roots grown in vitro. Amplification of juveniles (J2) from the starting inoculum is around 40fold. The method was validated for three Meloidogyne species (Meloidogyne javanica, $M$. incognita, and $M$. arenaria), producing viable and robust freshly hatched J2s. These J2s can be used for further in vitro infection of different plant species such as Arabidopsis, tobacco and tomato, as well as to maintain and amplify the population. The method allowed maintenance of around 90 Meloidogyne sp. generations (one every 2 months) from a single initial female over 15 years.

Keywords: amplification, Meloidogyne sp., root-knot nematodes, cucumber, in vitro culture, monoxenic

\section{INTRODUCTION}

Root-knot nematodes (RKNs; Meloidogyne sp.) constitute major pests in agriculture worldwide, causing annual economic losses estimated at $\$ 118$ billion, (McCarter, 2008). They are obligate parasites that penetrate plant roots to establish their feeding sites, called giant cells (GCs), causing thickenings or knots in the roots referred to as galls (Escobar et al., 2015). Meloidogyne javanica $(M j), M$. incognita $(M i)$, and M. arenaria $(M a)$ are the most common species of RKNs in the warm climate of southern Europe but also in glasshouses of the more temperate climate of northern Europe (Wesemael et al., 2011). The polyphagous behavior of RKNs as well as the ban on the most effective agrochemical nematicides constitutes a challenge for the successful management of this pest (Haydock et al., 2013). Understanding the molecular processes underlying the formation of 
galls and GCs and a deep knowledge of the nematode's biology are crucial for the development of new biotechnology-based control methods (reviewed in Fosu-Nyarko and Jones, 2015). Efficient nematode amplification and maintenance in the laboratory under aseptic experimental conditions are important and quite valuable for molecular biology techniques, such as transcriptomics (as massive sequencing or microarray hybridization), proteomics, metabolomics, etc., being free of biological contamination. So far, the usual practice is to surface-sterilize either eggs or nematode juveniles (J2s) from greenhouse-grown host plant specimens. Normally, a combination of different disinfection methods (mercuric chloride, chlorhexidine, streptomycin sulfate, bleach, antibiotics, physical filters, etc.; Huettel, 1990) is used; however, excessive doses of disinfectants may be toxic, leading to poor nematode survival, whereas insufficient dosage will not ensure efficient sterilization. In vitro culture presents the additional advantages of a reduced growth chamber space and non-daily maintenance. Therefore, several protocols for monoxenic nematode cultures have been developed: for example, in tomato excised roots or seedlings (Sayre, 1958; Mathur et al., 1980; Orion et al., 1980; Sudirman and Webster, 1995; Hutangura et al., 1998); in Abelmoschus esculentus (Tanda et al., 1980); in onion root cultures (Mitkowski and Abawi, 2002); in Agrobacterium rhizogenes-transformed roots of potato, tomato, bindweed, tropical tomato, lima bean, and carrot (Verdejo et al., 1988; Mitkowski and Abawi, 2002). Hydroponic or semihydroponic cultures have also been described (Atamian et al., 2012) but nematodes and plants were not completely aseptic.

Here, we describe a simple monoxenic culture method using cucumber roots (Cucumis sativus) to amplify different Meloidogyne spp. populations. This efficient method provides a viable and prolonged culture system for these obligate plant parasitic nematodes. The main proof of concept is that a population of $M$. javanica has been established from a single female and has been maintained in our laboratory for more than 15 years. The amplification has been successful at a ratio around $40 \mathrm{Pf} / \mathrm{Pi}$ (final population/initial population) from the initial $\mathrm{J} 2 \mathrm{~s}$ inoculum for each re-inoculation obtained. Moreover, J2s from these cultures can be used directly after a simple hatching step in aseptic conditions to infect plants for experiments with different in vitro grown plant species. Successful experiments have been performed with this procedure on different genotypes of Arabidopsis, tobacco, and tomato (Barcala et al., 2010; Escobar et al., 2010; Portillo et al., 2013; Cabrera et al., 2014, 2015, 2016).

\section{MATERIALS AND METHODS}

The monoxenic nematode culture on cucumber roots was initiated 15 years ago, when egg masses from soil-grown infected tomato plants were used (Figure 1). These egg masses were used to obtain sterilized eggs according to Verdejo-Lucas (1995) to inoculate the first pool of 21-day-old cucumber seedlings grown in vitro. Every month a new cucumber batch was inoculated with the J2s hatched from egg masses produced in cucumber roots that were inoculated 2 months before (Figure 1). A step-by-step protocol with references to all commercial products, materials and tips is provided as Supplemental Material.

\section{Plant Materials and Growth Conditions}

Fifty C. sativus (L.) cv. Hoffmanns Giganta seeds (Buzzy Seeds, Catalog Number: 02186) were surface-sterilized with $40 \mathrm{~mL}$ of undiluted commercial bleach (35 gr/L) for $45 \mathrm{~min}$ and subsequently washed five times with sterile distilled water under a laminar flow hood. Ten Petri dishes ( $14 \mathrm{~cm}$ diameter) containing modified Gamborg B5 solid media (see Supplemental Material) supplemented with $3 \%$ sucrose were used to sow five seeds/plate with sterile tweezers (Figure 1). Plates were sealed with one layer of Parafilm ${ }^{\circledR}$ first, then with Micropore ${ }^{\circledR}$ tape and finally covered with aluminum foil to favor the development of the root system in darkness and avoid contamination, as it should be a long-lasting axenic culture. After 2 days of stratification at $4^{\circ} \mathrm{C}$, the plates were transferred to a dark growth chamber at $26^{\circ} \mathrm{C}$ for 21 days (Figures 1 and 2a). Just before inoculation with J2s, the etiolated aerial parts of the cucumber seedlings were removed to promote further root growth.

\section{Nematode Inoculation}

Cucumber plates used to collect the egg masses (Figure 2a) had to be carefully checked under a stereo-microscope to detect any visible contamination. Egg masses selected for hatching should have an amber color (Figures $\mathbf{2 b , c}$ ) when they are around 2 months old. Darker brown egg masses (Figure 2d) should be avoided as they are old and will produce a lower number of less vigorous nematodes. It is recommended to observe some hatched J2 moving around the egg masses as this normally indicates that $\mathrm{J} 2$ are viable (Figure $2 \mathrm{c}$ ). When egg masses are old, several dead juveniles are frequently found around them (Figure 2d). Four days before inoculation, 50 sterile egg masses (from one or several previous plates of cucumber plants 2 months after inoculation; Figure 1) were placed in a hatching jar in a sterile cell strainer (with a $70 \mu \mathrm{m}$ nylon mesh) inside a beaker filled with $5 \mathrm{~mL}$ of sterile tap water (see Supplemental Material). The mesh retained the egg masses while the hatched J2s moved through the sieve and subsequently sank to the bottom of the beaker. Hatching took place in the dark at $26^{\circ} \mathrm{C}$ for 4 days, and $1 \mathrm{~mL}$ with the freshly hatched J2s was used to inoculate the cucumber plates every 23 days (Figure 1). In general, one Petri dish is enough to provide the 50 egg masses needed to inoculate the 10 new cucumber plates; the remaining nine infected cucumber plates in each batch can be used for hatching J2s for in vitro experiments (Figure 1). Plates were inoculated with $1 \mathrm{ml}$ from the hatching jar, comprising approximately 1000 J2 (Figure 2a, Supplemental Material). After J2 inoculation, the plates were double sealed, covered with aluminum foil and placed back into the growth chamber for 2 months (Figure 1) till lifecycle completion, when new egg masses were produced. These egg masses can be used again to obtain more juveniles for new cucumber seedling inoculation (Figure 1). Instead of freshly hatched J2s, egg masses from the infected cucumber seedlings could also be used to inoculate new plates. In this case, from three to five egg masses per plate were placed on the agar medium near the roots with 


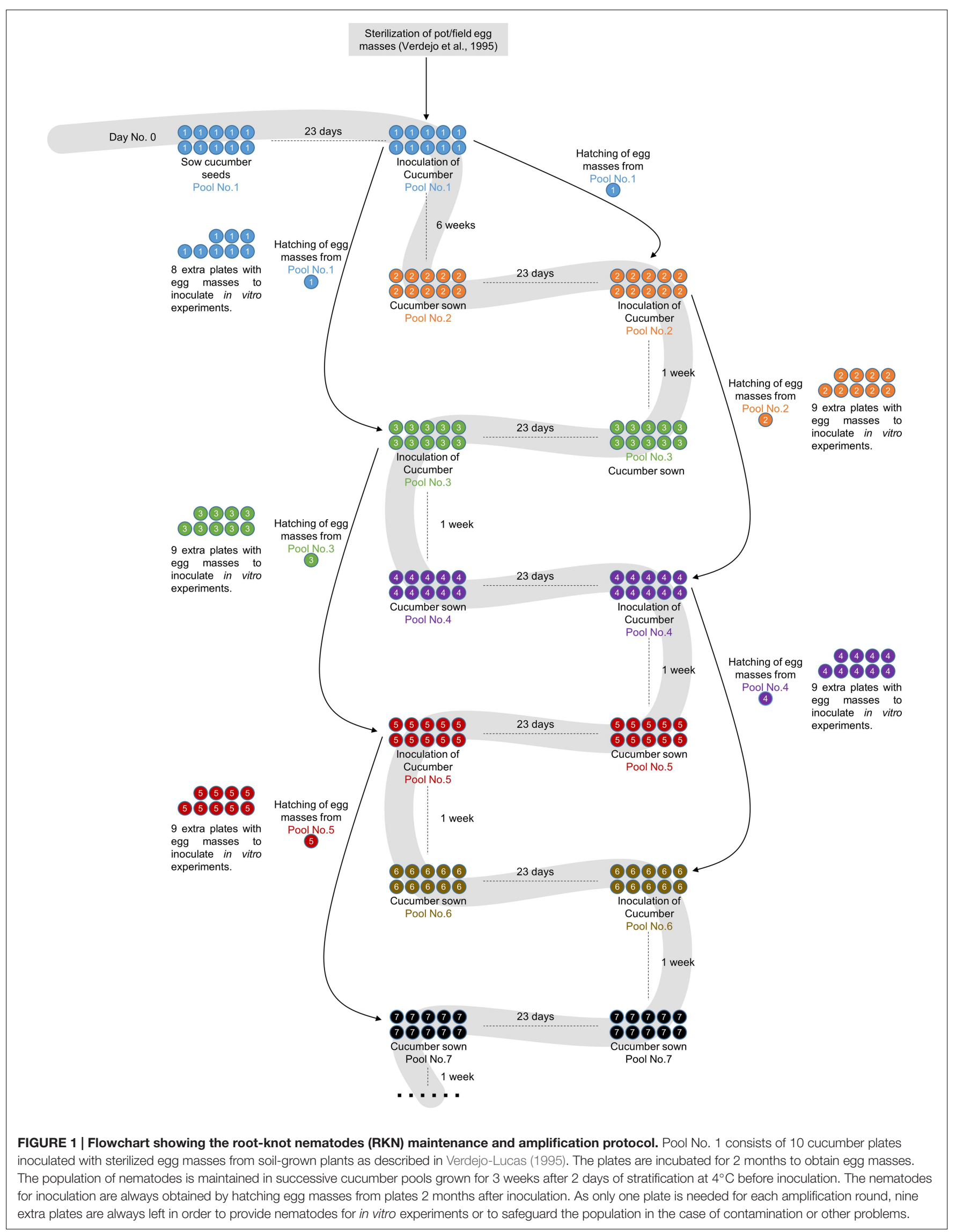




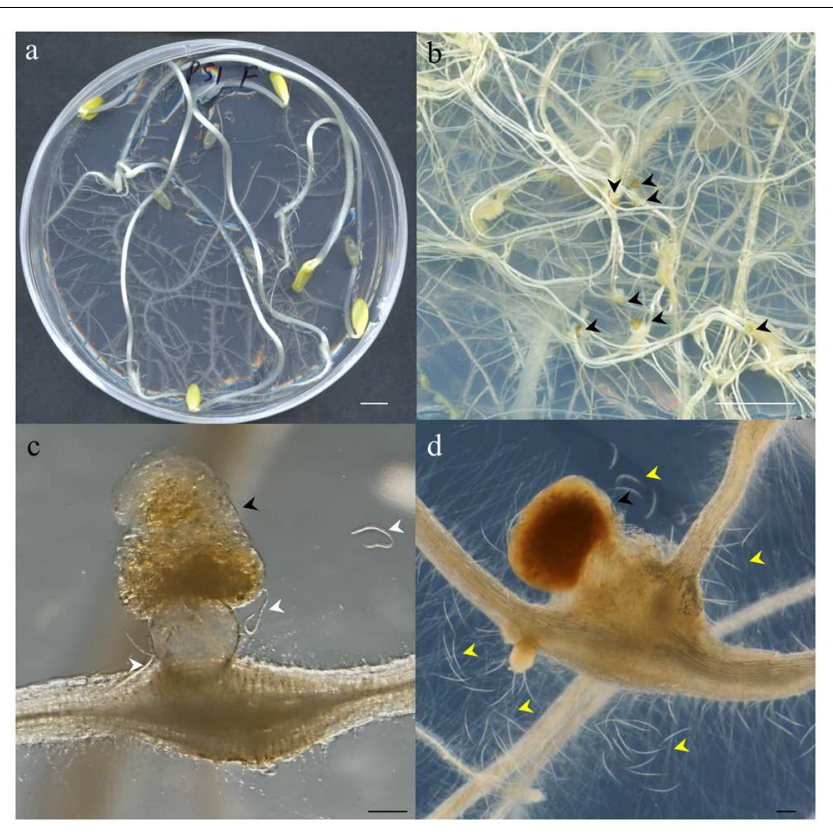

FIGURE 2 | Photographs illustrating different steps of the method described. (a) Etiolated Cucumis sativus seedlings 21 days after germination showing extensive root development before nematode inoculation. (b) Close-up of Meloidogyne javanica infected C. sativus roots showing the galls induced by this nematode and the egg masses deposited by the females outside the root (black arrowheads). (c) Close-up of a gall containing one female laying an egg mass with amber color in an optimum stage for hatching to obtain vigorous J2s. Hatched J2s around the egg mass are marked by white arrowheads. (d) Close-up of an unviable dark brown egg mass containing juveniles which are often dead. The dead J2s are easily observed around the egg mass and gall (yellow arrowheads). Scale bars: $1 \mathrm{~cm}(\mathbf{a}, \mathbf{b})$ and $200 \mu \mathrm{m}$ (c,d).

the help of sterile tweezers. However, this procedure can cause many asynchronous infections, as juveniles hatch gradually from the eggs. It is also important to point that if required, several amplification rounds can be performed from a plate infected with a single egg mass from a single female. This can be crucial in some experiments where clonal nematodes are required to reduce variability. Once the culture is stablished, we recommend to use the plates from this clonal amplification of females for further experiments and amplifications. It is advisable to settle a new stock of cucumber plates every 21 days, so that several different stock plates at different stages are generated (Figure 1).

\section{RESULTS AND DISCUSSION}

We determined the hatching ability of egg masses produced following the protocol described above for three different RKN species: $M j, M i$, and $M a$. Fifty egg masses (2-month-old) obtained from a single female clonal culture were collected for each species and incubated for 4 days in $5 \mathrm{~mL}$ of sterile tap water (see Supplemental Material) in a hatching jar. The number of J2 was assessed by counting under the stereo-microscope in three aliquots of $30 \mu \mathrm{l}$ each. The average J2 number among the three replicates was considered as a good estimation for the total number of hatched J2s. Mj egg masses yielded the highest number of J2s per egg mass and per $\mathrm{mL}(21.8 \mathrm{~J} 2 \mathrm{~s} / \mathrm{em} \cdot \mathrm{mL}$; Table 1). A slightly smaller number was obtained for $M a(19.7 \mathrm{~J} 2 \mathrm{~s} / \mathrm{em} \cdot \mathrm{mL})$ and $\mathrm{Mi}(17.9 \mathrm{~J} 2 \mathrm{~s} / \mathrm{em} \cdot \mathrm{mL}$; Figure 3A; Table 1). After the first hatching was collected, a new volume of $5 \mathrm{~mL}$ of sterile tap water was added to the hatching jar to favor a second hatching round from the same masses for another 4 days. The number of juveniles obtained in this second round was higher than in the first hatching for $M j$ and $M i$ (Figure 3A; Table 1; 25.3 and $24.3 \mathrm{~J} 2 \mathrm{~s} / \mathrm{em} \cdot \mathrm{mL}$, respectively), while the number was maintained for $\mathrm{Ma}$ (Figure 3A; Table 1). In a third hatching round, under the same conditions, the number of juveniles decreased in comparison with the first and second rounds for all species (Figure 3A; Table 1). Each hatching jar contained 50 egg masses in $5 \mathrm{~mL}$ of sterile distilled water, and in each hatching round an average of 5290, 4777, and $4625 \mathrm{~J} 2 \mathrm{~s}$ of $\mathrm{Mj}$, $M i$, and $M a$, respectively (Figure 3A; Table 1) were obtained that could be used for inoculation of plants grown in vitro. The sum of all three hatchings from the 50 egg masses of each species yielded a total number of 15869, 14330, and 13875 juveniles from $M j, M i$, and $M a$, respectively (Figure 3A; Table 1). As the nematodes came from a monoxenic culture, there was no need for chemical sterilization of juveniles. These treatments reduce their vigor and viability and usually results in a high variation in the infection ability of the J2s, ranging from very inefficient infection to wounding effects when too many nematodes tended to penetrate the same root. In contrast, $\mathrm{J} 2 \mathrm{~s}$ from the described aseptic culture were in the optimum infectivity state that did not vary much in different batches. This allowed the use of a reduced nematode inoculum (10 nematodes per plant) to avoid undesired root damage (Cabrera et al., 2014, 2015). Another advantage of the method herein described is that it allows for three independent biological replicates by infecting plants 4 days apart with $\mathrm{J} 2$ hatched from the same egg mass pool from three independent hatchings. This method also contributes to the homogeneity of the infection efficiency, reducing variability among experiments. All hatching data presented here are the average of more than 20 amplification rounds $(n=45$ for $M j ; n=20$ for $M i$ and $M a$ ) performed during the last 5 years (10 years after the initial inoculation). It is important to point that the amplification ability of the population may have changed since the first set of infections took place 15 years ago.

Juveniles from $M j, M i$, and $M a$ obtained from monoxenic cultures were used to inoculate plates containing five etiolated cucumber seedlings 23 days after sowing. Two months after inoculation, the number of egg masses developed in each plate was counted under a stereo-microscope and, subsequently, the "No. of egg masses per $500 \mathrm{~J} 2 \mathrm{~s}$ of the initial inoculum" was calculated (Table 1; Figure 3B). Each plate was inoculated with $1 \mathrm{~mL}$ of sterile tap water containing J2s from the hatching jar from any of the hatching rounds (see Table 1 for nematode numbers). Thus, the average number of $\mathrm{J} 2$ in the inoculum was 1058, 955, and 925 for $M j, M i$, and $M a$, correspondingly (Table 1). With this inoculum, it was possible to obtain an 


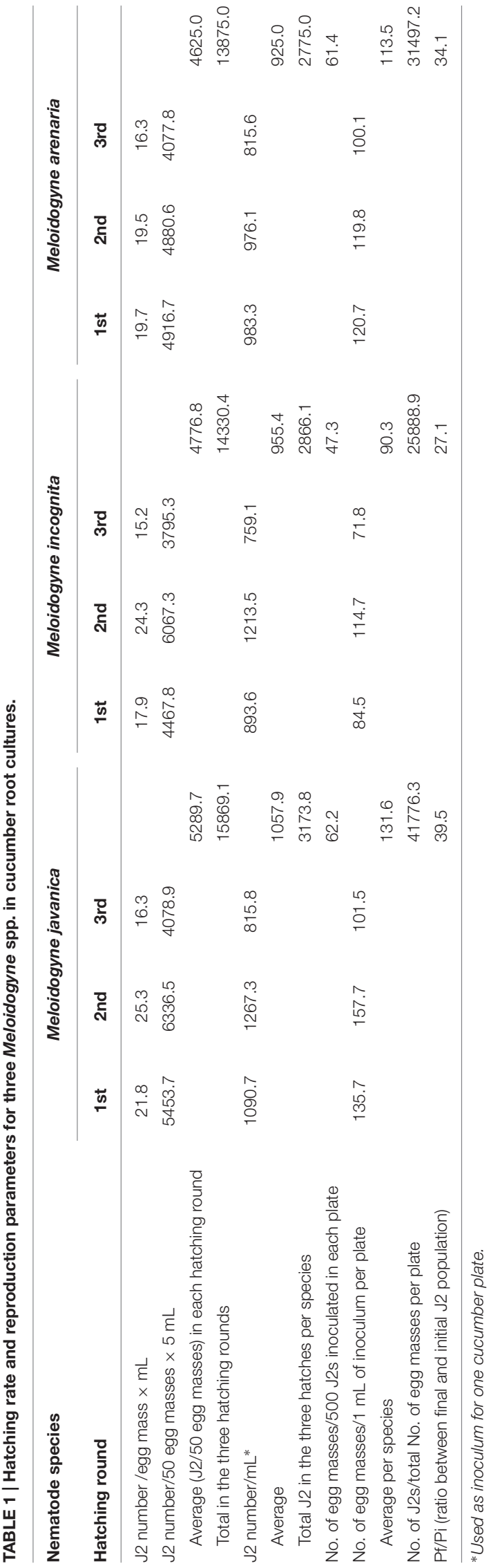

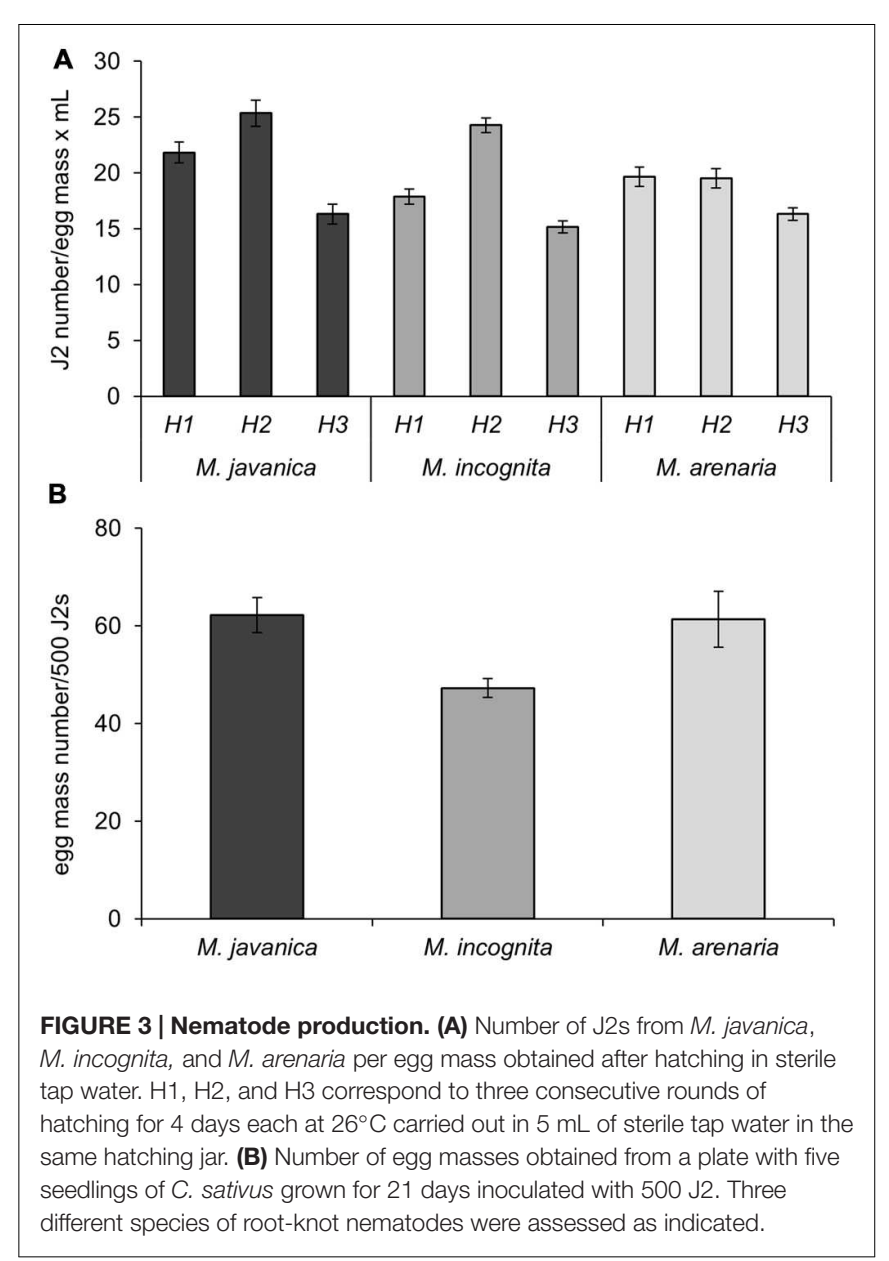

average of 132, 90, and 113 egg masses for each species in each plate (Table 1). $M j$ and $M a$ juveniles seemed to be the species with the greatest capacity to reproduce in vitro in cucumber roots as 62 and 61 egg masses were obtained in each plate per $500 \mathrm{~J} 2$ from the initial inoculum (Table 1; Figure 3B). The number of egg masses obtained from $\mathrm{Mi}$ was slightly smaller; 47 egg masses per plate inoculated with $500 \mathrm{~J} 2$ (Figure 3B). All three species reproduced efficiently in cucumber roots, however, $M j$ showed the best hatching and reproduction parameters in our system (Figure 3; Table 1). The number of juveniles from any of the three species could be increased ten-fold from the initial inoculum when 10 plates with five cucumber seedlings were used. Depending on the number of hatched J2 obtained per each egg mass, it is possible to obtain a total number of 41776, 25888, and $31497 \mathrm{~J} 2 \mathrm{~s}$ for $M j, M i$, and $M a$ (Table 1), respectively, from all the egg masses produced in a single cucumber plate. Data from tomato roots transformed with $A$. rhizogenes in vitro infected with an aggressive M. hapla population indicated a production of 20000 nematodes per plate after 8 weeks of growth (Mitkowski and Abawi, 2002). Regardless of the different Meloidogyne sp. we obtained for all three species a higher amplification rate in a similar period. Moreover, for $M j$ it almost doubled that of $M$. hapla in transformed roots. Comparison with amplification 
methods in soil are precluded, as the plant growing conditions also influence nematode reproduction.

The amplification ratio from the initial $\mathrm{J} 2$ population used for inoculation (Pf/Pi) was $39.5(\mathrm{Mj}), 27.1(\mathrm{Mi})$, and $34.1(\mathrm{Ma})$ (Table 1). This is in the range obtained with $A$. rhizogenes transformed roots from bindweed, bean, carrot and tropical tomato for M. javanica, but lower than the amplification obtained in potato and tomato (Solanum lycopersicum Mill. cv. South Australian Early Dwarf Red) transgenic roots $(\mathrm{Pf} / \mathrm{Pi}=83$ and 161, respectively; Verdejo et al., 1988). Although, this last method based on in vitro infected transgenic roots is sufficient to amplify Meloidogyne spp. in monoxenic cultures, the method based on cucumber roots is simpler, as it does not require root transformation. When cucumber root plates get contaminated, a new batch of seeds can be easily germinated. On the other hand, when hairy root systems are used, extensive contamination of transformed roots plates may require a new transformation event.

Here, we report the use of cucumber as a suitable host for Meloidogyne spp. maintenance in monoxenic cultures. We have been able to amplify different Meloidogyne spp. over 15 years routinely in the laboratory. Cucumber can be easily cultivated in vitro as its seedlings develop a dense root system within a short time. Thus, amplification of $M j, M i$, and $M a$ populations of at least 39.5, 27.1, and 34.1 times, respectively, was achieved from the initial $\mathrm{J} 2$ inoculum. The differences in the amplification ratio among the different species are probably caused by diverse virulence of the nematode populations used (Semblat et al., 2000).

Finally, our system is suitable as it is low-cost and no timeconsuming as well as it requires basic laboratory equipment. In addition, it is easily restored in case of in vitro culture contamination or an accidental heat shock or other problems caused by growth chamber failure. Our main proof of the suitability of the method is that a population of $M j$ started from a single female and was maintained in a cucumber monoxenic culture in our laboratory after following the protocol presented here for more than 15 consecutive years.

\section{REFERENCES}

Atamian, H. S., Roberts, P. A., and Kaloshian, I. (2012). High and low throughput screens with Root-Knot Nematodes Meloidogyne spp. J. Vis. Exp. 61:e3629. doi: $10.3791 / 3629$

Barcala, M., García, A., Cabrera, J., Casson, S., Lindsey, K., Favery, B., et al. (2010). Early transcriptomic events in microdissected Arabidopsis nematodeinduced giant cells. Plant J. 61, 698-712. doi: 10.1111/j.1365-313X.2009.04 098.x

Cabrera, J., Barcala, M., García, A., Rio-Machín, A., Medina. C., JaubertPossamai, S., et al. (2016). Differentially expressed small RNAs in Arabidopsis galls formed by Meloidogyne javanica: a functional role for miR390 and its TAS3-derived tasiRNAs. New Phytol. 209, 1625-1640. doi: 10.1111/nph.13735

Cabrera, J., Díaz-Manzano, F. E., Barcala, M., Arganda-Carreras, I., AlmeidaEngler, J., Engler, G., et al. (2015). Phenotyping nematode feeding sites: three-dimensional reconstruction and volumetric measurements of giant cells induced by root-knot nematodes in Arabidopsis. New Phytol. 206, 868-880. doi: 10.1111/nph.13249

Cabrera, J., Díaz-Manzano, F. E., Sánchez, M., Rosso, M. N., Melillo, T., Goh, T., et al. (2014). A role for LATERAL ORGAN BOUNDARIES-DOMAIN 16 during the interaction Arabidopsis-Meloidogyne spp. provides a molecular link

\section{AUTHOR CONTRIBUTIONS}

All authors substantially contributed to the acquisition (FEDM, $\mathrm{RO}, \mathrm{MB}$, JC), analysis (FEDM, JC, CE) or interpretation (FEDM, RO, MB, JC, CE, CF) of the data presented in this manuscript. All authors drafted and approved the manuscript. All authors are accountable for all aspects of the work and ensure that questions related to the accuracy or integrity of any part of the work are appropriately investigated and resolved.

\section{FUNDING}

This work was supported by the Spanish government (grant AGL2013-48787 to CE, and CSD2007-057 and PCIN-2013053 to CF) and by the Castilla-La Mancha government (PEII2014-020-P to CF). JC, RO, and FD-M were supported by a fellowships from the Ministry of Education, Science and Technology.

\section{ACKNOWLEDGMENTS}

We acknowledge Jose Escobar's help with acquisition of the egg mass photograph in Figure 2c. We also acknowledge María Sanchez, Alejandra García, Mary Portillo, Ana Belén Yuste, and Ana Rapp for maintaining over the years Meloidogyne sp. populations following the protocol presented here.

\section{SUPPLEMENTARY MATERIAL}

The Supplementary Material for this article can be found online at: http://journal.frontiersin.org/article/10.3389/fpls.2016. 00124

between lateral root and root-knot nematode feeding site development. New Phytol. 203, 632-645. doi: 10.1111/nph.12826

Escobar, C., Barcala, M., Cabrera, J., and Fenoll, C. (2015). "Overview of Root-knot nematodes and giant cells," in Advances in Botanical Research, Vol. 73, eds C. Escobar and C. Fenoll (Oxford: Elsevier Academic Press), 1-32.

Escobar, C., García, A., Aristizábal, F., Portillo, M., Herreros, E., Muñoz-Martín, M. A., et al. (2010). Activation of geminivirus V-sense promoters in roots is restricted to nematode feeding sites. Mol. Plant Pathol. 11, 409-417. doi: 10.1111/j.1364-3703.2010.00611.x

Fosu-Nyarko, J., and Jones, M. G. K. (2015). "Application of biotechnology for nematode control in crop plants," in Advances in Botanical Research, Vol. 73, eds C. Escobar and C. Fenoll (Oxford: Elsevier Academic Press), 339-376.

Haydock, P. P. J., Woods, S. R., Grove, I. G., and Hare, M. C. (2013). "Chemical control of nematodes," in Plant Nematology, eds R. N. Perry and M. Moens (Wallingord: CABI), 259-279.

Huettel, R. N. (1990). "Monoxenic culturing of plant parasitic nematodes using carrot disks, callus tissue and root-explants," in Plant Nematology Laboratory Manual, eds B. M. Zuckermann, W. F. Mai, and L. R. Krusberg (Amherst, MA: Agricultural Experiment Station, University of Massachusetts), 163-172.

Hutangura, P., Jones, M. G. K., and Heinrich, T. (1998). Optimisation of culture conditions for in vitro infection of tomato with the Root-Knot Nematode 
Meloidogyne javanica. Australas. Plant Pathol. 27, 84-89. doi: 10.1071/AP 98010

Mathur, A., Mohan, J., and Kant, U. (1980). Growth and phenolic contents of root gall and normal tissues of Lycopersicon esculentum in tissue culture. Indian J. Mycol. Plant Pathol. 10:44.

McCarter, J. P. (2008). "Molecular approaches toward resistance to plant-parasitic nematodes," in Cell Biology of Plant Nematode Parasitism, eds R. H. Berg and C. G. Taylor (Berlin: Springer), 239-267.

Mitkowski, N., and Abawi, G. (2002). Monoxenic maintenance and reproduction of root-knot nematode (Meloidogyne hapla) on multiple-species in vitro root culture systems. Plant Cell Rep. 21, 14-23. doi: 10.1007/s00299-0020468-6

Orion, D., Wergin, W. P., and Endo, B. Y. (1980). Inhibition of syncytia formation and root-knot nematode development on cultures of excised tomato roots. J. Nematol. 12, 196-203.

Portillo, M., Cabrera, J., Lindsey, K., Topping, J., Andrés, M. F., Ememiliozzi, M., et al. (2013). Distinct and conserved transcriptomic changes during nematodeinduced giant cell development in tomato compared with Arabidopsis: a functional role for gene repression. New Phytol. 197, 1276-1290. doi: 10.1111/nph.12121

Sayre, R. M. (1958). Plant Tissue Culture as a Tool in the Physiology of Root-Knot Nematode, Meloidogyne Incognita, Ph. D. Dissertation, University of Nebraska, Lincoln.

Semblat, J. P., Bongiovanni, M., Wajnberg, E., Dalmasso, A., Abad, P., and Castagnone-Sereno, P. (2000). Virulence and molecular diversity of parthenogenetic root-knot nematodes. Meloidogyne spp. Heredity 84, 81-89. doi: 10.1046/j.1365-2540.2000.00633.x
Sudirman, and Webster, J. M. (1995). Effect of ammonium ions on egg hatching and second-stage juveniles of Meloidogyne incognita in axenic tomato root culture. J. Nematol. 27, 346-352.

Tanda, A., Atwal, A., and Bajaj, Y. (1980). Reproduction and maintenance of RootKnot Nematode (Meloidogyne incognita) in excised roots and callus cultures of okra (Abelmoschus esculentus). Indian J. Exp. Biol. 18, 1340-1342.

Verdejo, S., Jaffee, B. A., and Mankau, R. (1988). Reproduction of Meloidogyne javanica on plant roots genetically transformed by Agrobacterium rhizogenes. J. Nematol. 20, 599-604.

Verdejo-Lucas, S. (1995). "Dual culture: nematodes," in Molecular Methods in Plant Pathology, eds R. P. Sing and U. S. Sing (London: CRC Lewis Publisher), 301-312.

Wesemael, W. M., Viaene, N., and Moens, M. (2011). Root-knot nematodes (Meloidogyne spp.) in Europe. Nematology 13, 3-16. doi: $10.1163 / 138855410 \times 526831$

Conflict of Interest Statement: The authors declare that the research was conducted in the absence of any commercial or financial relationships that could be construed as a potential conflict of interest.

Copyright (c) 2016 Díaz-Manzano, Olmo, Cabrera, Barcala, Escobar and Fenoll. This is an open-access article distributed under the terms of the Creative Commons Attribution License (CC BY). The use, distribution or reproduction in other forums is permitted, provided the original author(s) or licensor are credited and that the original publication in this journal is cited, in accordance with accepted academic practice. No use, distribution or reproduction is permitted which does not comply with these terms. 